Georgian Mathematical Journal

Volume 11 (2004), Number 3, 489-494

\title{
ON THE MEASURABILITY OF FUNCTIONS WITH RESPECT TO CERTAIN CLASSES OF MEASURES
}

\author{
A. KHARAZISHVILI AND A. KIRTADZE
}

\begin{abstract}
The concept of measurability of real-valued functions with respect to various classes of measures is introduced and the associated notion of an absolutely nonmeasurable function is investigated. A characterization of such functions is given. Also, it is shown that functions produced by the classical Vitali partition of the real line are measurable with respect to the class of all extensions of the Lebesgue measure on this line.
\end{abstract}

2000 Mathematics Subject Classification: 28A05, 28D05.

Key words and phrases: Absolutely nonmeasurable function, generalized Luzin set, Vitali partition, extension of measure.

Let $E$ be a set and let $M$ be a class of measures on $E$ (we assume, in general, that the domains of measures from $M$ are various $\sigma$-algebras of subsets of $E$ ). We say that a real-valued function $f: E \rightarrow \mathbf{R}$ is measurable with respect to $M$ if there exists at least one measure $\mu \in M$ such that $f$ is measurable with respect to $\mu$. Otherwise, we say that $f$ is absolutely nonmeasurable with respect to $M$.

Let $\mu$ be a measure on $E$. As usual, we say that $\mu$ is diffused (or continuous) if it vanishes on all singletons in $E$.

Example 1. For any set $E$, let $M_{E}$ be the class of all nonzero $\sigma$-finite diffused measures on $E$. Let $f: E \rightarrow \mathbf{R}$ be a function and let, for some $t_{0} \in \mathbf{R}$, the relation $\operatorname{card}\left(f^{-1}\left(t_{0}\right)\right)>\omega$ be satisfied, where $\omega$ denotes the first infinite cardinal number. In this case, we can assert that $f$ is measurable with respect to $M_{E}$. Indeed, it is not difficult to define a complete diffused probability measure $\mu$ on $E$ such that $\mu\left(f^{-1}\left(t_{0}\right)\right)=1$. Consequently, for any set $T \subset \mathbf{R}$, we have $\mu\left(f^{-1}(T)\right)=1$ if $t_{0} \in T$, and $\mu\left(f^{-1}(T)\right)=0$ if $t_{0} \notin T$. This, obviously, implies that $f$ is measurable with respect to the measure $\mu$ (hence, with respect to the class $\left.M_{E}\right)$.

In particular, if an original set $E$ is such that $\operatorname{card}(E)>2^{\omega}$, then every function $f: E \rightarrow \mathbf{R}$ is measurable with respect to $M_{E}$.

Let $P$ be a topological space whose all singletons belong to the Borel $\sigma$ algebra of $P$. We recall that $P$ is a universal measure zero space if there exists no nonzero $\sigma$-finite diffused Borel measure on $P$. It is well known that there are uncountable universal measure zero subspaces of the real line $\mathbf{R}$. One classical construction of such a subspace of $\mathbf{R}$ is due to Luzin and is presented, e.g., in $[1]$. 
Taking into account Example 1 and using the notion of a universal measure zero space, we can obtain a characterization of absolutely nonmeasurable functions (with respect to the class $M_{E}$ ).

Theorem 1. A function $f: E \rightarrow \mathbf{R}$ is absolutely nonmeasurable with respect to $M_{E}$ if and only if the following two conditions hold:

1) for each $r \in \mathbf{R}$, the set $f^{-1}(r)$ is at most countable;

2) the set $\operatorname{ran}(f)$ (i.e., the range of $f$ ) is a universal measure zero subspace of $\mathbf{R}$.

Proof. Suppose first that $f$ is absolutely nonmeasurable with respect to $M_{E}$. Then the argument given in Example 1 shows that condition 1) must be satisfied. Let us verify that condition 2) must be valid, too. Indeed, assuming that $\operatorname{ran}(f)$ is not a universal measure zero subset of $\mathbf{R}$, consider some Borel diffused probability measure $\nu$ on $\operatorname{ran}(f)$ and denote

$$
\mathcal{S}=\left\{f^{-1}(Z): Z \in \operatorname{dom}(\nu)\right\}
$$

Clearly, $\mathcal{S}$ is a $\sigma$-algebra of subsets of $E$ and the family of countable sets $\left\{f^{-1}(r): r \in \operatorname{ran}(f)\right\}$ forms a partition of $E$. We put

$$
\mu\left(f^{-1}(Z)\right)=\nu(Z) \quad(Z \in \operatorname{dom}(\nu)) .
$$

In this manner, the diffused probability measure $\mu$ on the $\sigma$-algebra $\mathcal{S}$ is defined so that $f$ turns out to be measurable with respect to $\mu$. However, this contradicts our assumption that $f$ is absolutely nonmeasurable with respect to $M_{E}$. The contradiction obtained shows the necessity of conditions 1) and 2) for the absolute nonmeasurability of $f$ with respect to $M_{E}$.

Now, suppose that these two conditions are fulfilled for $f$ and let us establish that $f$ is absolutely nonmeasurable with respect to $M_{E}$. Suppose for a moment that there exists a measure $\mu$ belonging to $M_{E}$ such that $f$ is measurable with respect to $\mu$. We may assume, without loss of generality, that $\mu$ is a probability measure. Then, denoting by $\mathcal{B}(\operatorname{ran}(f))$ the Borel $\sigma$-algebra of $\operatorname{ran}(f)$, we may define

$$
\nu(Z)=\mu\left(f^{-1}(Z)\right) \quad(Z \in \mathcal{B}(\operatorname{ran}(f))) .
$$

So we get a Borel probability measure $\nu$ on $\operatorname{ran}(f)$ which is diffused in view of condition 1). But this contradicts condition 2). The contradiction obtained ends the proof of the theorem.

Remark 1. Let $\omega_{1}$ denote, as usual, the first uncountable cardinal number. It is impossible to prove (within ZFC theory) the existence of absolutely nonmeasurable functions with respect to the class $M_{E}$ where $\operatorname{card}(E)>\omega_{1}$. This fact directly follows from Theorem 1 and the circumstance that there are models of set theory (constructed by Baumgartner and Laver) in which the cardinality of any universal measure zero subspace of $\mathbf{R}$ does not exceed $\omega_{1}$.

Assuming some additional set-theoretical axioms, it is not difficult to demonstrate that there exists an absolutely nonmeasurable function $f: \mathbf{R} \rightarrow \mathbf{R}$ with respect to the class $M_{\mathbf{R}}$. For instance, the existence of such a function follows 
from the existence of a Luzin subset of $\mathbf{R}$ (detailed information about Luzin sets can be found, e.g., in [2]). Moreover, the next example shows that absolutely nonmeasurable functions can be found even among injective homomorphisms of the additive group $\mathbf{R}$ into itself.

Example 2. Under Martin's Axiom, there exists a generalized Luzin set $X \subset \mathbf{R}$ being simultaneously a vector space over the field $\mathbf{Q}$ of all rational numbers (the construction of such a set $X$ is fairly standard, by using the method of transfinite recursion). It is well known that every Luzin set is a universal measure zero space and, under Martin's Axiom, every generalized Luzin set is a universal measure zero space, too. In particular, $X$ is a universal measure zero subset of $\mathbf{R}$. Let $g: \mathbf{R} \rightarrow X$ denote some isomorphism between $\mathbf{R}$ and $X$ both of which are regarded as vector spaces over $\mathbf{Q}$. Then $g$ can be considered as an injective group homomorphism from $\mathbf{R}$ into $\mathbf{R}$ and therefore $g$ is a nontrivial solution of the classical Cauchy functional equation

$$
\phi(x+y)=\phi(x)+\phi(y) \quad(x \in \mathbf{R}, \quad y \in \mathbf{R}) .
$$

Furthermore, according to Theorem 1, g turns out to be absolutely nonmeasurable with respect to the class $M_{\mathbf{R}}$.

In this context, let us recall that any nontrivial solution of the Cauchy functional equation is necessarily nonmeasurable in the Lebesgue sense.

Let $S$ be an equivalence relation on $\mathbf{R}$ whose all equivalence classes are at most countable. We shall say that $f: \mathbf{R} \rightarrow \mathbf{R}$ is a Vitali type function for $S$ if $(r, f(r)) \in S$ for each $r \in \mathbf{R}$ and the set $\operatorname{ran}(f)$ is a selector of the partition of $\mathbf{R}$ determined by $S$.

It is widely known that if $V$ is the classical Vitali equivalence relation on $\mathbf{R}$ (i.e., $(x, y) \in V \Leftrightarrow x-y \in \mathbf{Q}$ ), then any Vitali type function for $V$ is absolutely nonmeasurable with respect to the class of all translation-invariant extensions of the Lebesgue measure $\lambda$ on $\mathbf{R}$ (see, e.g., [2], [3], [4] or [5]). However, the following somewhat surprising statement is valid.

Theorem 2. Let $M(\lambda)$ denote the class of all measures on $\mathbf{R}$ extending $\lambda$. Then every Vitali type function for $V$ is measurable with respect to $M(\lambda)$.

Proof. Our argument is essentially based on one useful result of measure theory, stating that if $E$ is a set, $\mu$ is a $\sigma$-finite measure on $E$ and $\left\{X_{n}: n<\omega\right\}$ is a disjoint countable family of subsets of $E$, then there always exists a measure $\mu^{\prime}$ on $E$ extending $\mu$ and satisfying the relation $\left\{X_{n}: n<\omega\right\} \subset \operatorname{dom}\left(\mu^{\prime}\right)$ (in this connection, see [6] and [7]).

Let $f: \mathbf{R} \rightarrow \mathbf{R}$ be a Vitali type function for $V$. Denote $X=\operatorname{ran}(f)$. The family of sets $\{X+q: q \in \mathbf{Q}\}$ forms a countable partition of $\mathbf{R}$. Therefore, according to the above-mentioned result, there exists a measure $\lambda^{\prime}$ on $\mathbf{R}$ extending $\lambda$ and such that $X+q \in \operatorname{dom}\left(\lambda^{\prime}\right)$ for all $q \in \mathbf{Q}$. We assert that $f$ is measurable with respect to $\lambda^{\prime}$. Indeed, for each Borel subset $B$ of $\mathbf{R}$, the equality

$$
f^{-1}(B)=\cup\{X \cap B+q: q \in \mathbf{Q}\}
$$


is easily verified. This equality can also be rewritten as

$$
f^{-1}(B)=\cup\{(X+q) \cap(B+q): q \in \mathbf{Q}\} .
$$

The right-hand side of the latter relation indicates that the set $f^{-1}(B)$ is $\lambda^{\prime}$ measurable, which yields at once the measurability of $f$ with respect to $\lambda^{\prime}$ (hence, with respect to $M(\lambda)$ ). This ends the proof.

Remark 2. If $\mu$ is a nonzero $\sigma$-finite diffused measure on $E$ and $\left\{X_{n}: n<\omega\right\}$ is a countable family of subsets of $E$, then, in general, we cannot assert that there exists a measure $\mu^{\prime}$ on $E$ extending $\mu$ and satisfying the relation $\left\{X_{n}: n<\right.$ $\omega\} \subset \operatorname{dom}\left(\mu^{\prime}\right)$. Moreover, if $E$ is of cardinality $\omega_{1}$, then there exists a countable family $\left\{X_{n}: n<\omega\right\}$ of subsets of $E$ such that for every nonzero $\sigma$-finite diffused measure $\mu$ on $E$ at least one set $X_{n}$ is nonmeasurable with respect to $\mu$.

Example 3. Assuming Martin's Axiom, it is not difficult to construct a generalized Luzin set $X \subset \mathbf{R}$ and an equivalence relation $S \subset \mathbf{R} \times \mathbf{R}$ such that:

1) $(\forall r \in \mathbf{R})(\operatorname{card}(S(r))=\omega)$;

2) $X$ is a selector of the partition $\{S(r): r \in \mathbf{R}\}$ of $\mathbf{R}$.

Let $h: \mathbf{R} \rightarrow \mathbf{R}$ be a Vitali type function for $S$ such that $\operatorname{ran}(h)=X$. Then, in view of Theorem $1, h$ is absolutely nonmeasurable with respect to the class $M_{\mathbf{R}}$.

The preceding example shows that the validity of Theorem 2 is essentially implied by some special (in fact, group-theoretical) properties of the classical Vitali partition of $\mathbf{R}$.

Example 4. Denote by $M^{\prime}(\lambda)$ the class of all those measures on $\mathbf{R}$ which extend $\lambda$ and are quasi-invariant under the group of all translations of $\mathbf{R}$. It can be demonstrated that there exists a Vitali type function for $V$ which is measurable with respect to $M^{\prime}(\lambda)$ (cf. [4] and [5]). At the same time, we do not know whether there exists a Vitali type function for $V$ absolutely nonmeasurable with respect to $M^{\prime}(\lambda)$.

Let $E$ be a set equipped with a $\sigma$-finite measure $\mu$ and let $f: E \rightarrow \mathbf{R}$ be a function satisfying the following condition: there exists a Borel probability measure $\nu$ on $\operatorname{ran}(f)$ such that the graph of $f$ is a $(\mu \times \nu)$-thick subset of the product space $E \times \operatorname{ran}(f)$.

Then, applying the standard argument (cf. [4] or [5]), it is not difficult to demonstrate that there exists a measure $\mu^{\prime}$ on $E$ such that:

1) $\mu^{\prime}$ extends $\mu$;

2) $f$ is measurable with respect to $\mu^{\prime}$.

In other words, $f$ turns out to be measurable with respect to the class $M(\mu)$ of all extensions of $\mu$.

In particular, if $E=\mathbf{R}$ and a function $f: \mathbf{R} \rightarrow \mathbf{R}$ has the $(\lambda \times \lambda)$-thick graph, then $f$ turns out to be measurable with respect to an appropriate extension of $\lambda$ (and thus $f$ is measurable with respect to the class $M(\lambda)$ ). Note that there are various examples of functions $f: \mathbf{R} \rightarrow \mathbf{R}$ whose graphs are $(\lambda \times \lambda)$-thick subsets of the plane $\mathbf{R}^{2}$ (see, e.g., [5] and [8]). Moreover, the following much stronger statement is valid. 
Theorem 3. There exists a function $g: \mathbf{R} \rightarrow \mathbf{R}$ having the property that, for any $\sigma$-finite diffused Borel measure $\mu$ on $\mathbf{R}$ and for any $\sigma$-finite measure $\nu$ on $\mathbf{R}$, the graph of $g$ is a $(\mu \times \nu)$-thick subset of the plane $\mathbf{R}^{2}$.

Proof. We start with a partition $\left\{X_{t}: t \in \mathbf{R}\right\}$ of $\mathbf{R}$ into Bernstein sets (recall that a Bernstein set is any totally imperfect subset of $\mathbf{R}$ whose complement is also totally imperfect). The existence of such a partition is well known (see, e.g., [1], [2], [5]). Define the required function $g$ as follows: for each $x \in \mathbf{R}$, put $g(x)=t$ if and only if $x \in X_{t}$.

Let $\mu$ be any $\sigma$-finite diffused Borel measure on $\mathbf{R}$ and let $\nu$ be an arbitrary nonzero $\sigma$-finite measure on $\mathbf{R}$. Let us verify that the graph of $g$ is $(\mu \times \nu)$-thick in $\mathbf{R}^{2}$. Indeed, if $Z$ is any $(\mu \times \nu)$-measurable set with $(\mu \times \nu)(Z)>0$, then, in virtue of the Fubini theorem, there exists a point $t_{0} \in \mathbf{R}$ such that

$$
\mu\left(\left\{x \in \mathbf{R}:\left(x, t_{0}\right) \in Z\right\}\right)>0 .
$$

Keeping in mind that $X_{t_{0}}$ is a Bernstein subset of $\mathbf{R}$, we get

$$
X_{t_{0}} \cap\left\{x \in \mathbf{R}:\left(x, t_{0}\right) \in Z\right\} \neq \varnothing .
$$

Therefore the graph of $g$ has the nonempty intersection with $Z$. This completes the proof.

\section{REFERENCES}

1. K. Kuratowski, Topology. I. Academic Press, New York-London; Państwowe Wydawnictwo Naukowe, Warsaw, 1966.

2. J. C. Oxтовy, Measure and category. A survey of the analogies between topological and measure spaces. Graduate Texts in Mathematics, 2. Springer-Verlag, New York-Berlin, 1971.

3. I. P. Natanson, Theory of functions of real variable. Second edition, revised. (Russian) Gosudarstv. Izdat. Tehn.-Teoret. Lit., Moscow, 1957.

4. A. B. Kharazishvili, Applications of point set theory in real analysis. Mathematics and its Applications, 429. Kluwer Academic Publishers, Dordrecht, 1998.

5. A. B. Kharazishvili, Strange functions in real analysis. Marcel Dekker, Inc., New YorkBasel, 2000.

6. D. BIERLEIN, Über die Fortsetzung von Wahrscheinlichkeitsfeldern. Z. Wahrscheinlichkeitstheorie und Verw. Gebiete 1(1962/1963), 28-46.

7. A. Ascherl and J. Lehn, Two principles for extending probability measures. Manuscripta Math. 21(1977), No. 1, 43-50.

8. B. R. Gelbaum and J. M. H. Olmsted, Counterexamples in analysis. The Mathesis Series, Holden-Day, Inc., San Francisco, Calif.-London-Amsterdam, 1964.

(Received 22.09.2003) 
Author's addresses:

A. Kharazishvili

I. Vekua Institute of Applied Mathematics

I. Javakhishvili Tbilisi State University

2, University St., Tbilisi 0143

Georgia

E-mail: kharaz@saba.edu.ge

A. Kirtadze

Department of Mathematics No. 63

Georgian Technical University

77, Kostava St., Tbilisi 0175

Georgia 